\title{
Anti-CD157 Monoclonal Antibody MEN1112
}

National Cancer Institute

\section{Source}

National Cancer Institute. Anti-CD157 Monoclonal Antibody MEN1112. NCI Thesaurus.

Code C120317.

A humanized, Fc engineered, de-fucosylated monoclonal immunoglobulin G1 (IgG1) antibody directed against the bone marrow stromal cell antigen 1 (BST 1/CD157), with potential antineoplastic activity. Upon intravenous infusion, anti-CD157 monoclonal antibody MEN1112 specifically binds to and induces an antibody dependent cell cytotoxic (ADCC) response against CD157-expressing tumor cells. CD157, also known as ADPribosyl cyclase 2, is a glycosyl-phosphatidylinositol (GPI)-anchored transmembrane protein belonging to the ADP-ribosyl-cyclase family and is overexpressed on certain cancer cell types. Fc-optimization of MEN1112, which involves the removal of fucose residues from its Fc domain, allows for enhanced Fc-gamma receptor binding on effector cells, such as natural killer (NK) cells, and further enhances tumor cell lysis. 